\title{
Alice Munro: Nobelpreisgekrönte Meisterin der Short Story aus Kanada
}

\section{Alice Munro und der Literaturnobelpreis}

Am 10. Oktober 2013 wurde verkündet, was der Literaturkritiker Denis Scheck, befürwortend, eine "sensationelle Wahl” nannte, was andere Fachleute aber durchaus für möglich, weil mehr als angemessen, angesehen hatten: der Literaturnobelpreis 2013 ging an die kanadische Short Story Autorin Alice Munro. Wenn man den kurzen Video-Clip auf der Webseite des Nobelpreiskomitees anschaut, lässt sich nach der Verkündigung durch den ständigen Sekretär des Nobelpreiskomitees die unmittelbare Reaktion der vor Ort Versammelten deutlich hören: beifallartige Rufe der Zustimmung, ja der Begeisterung. Diese spontane, sehr positive Reaktion der versammelten Journalisten bei der Bekanntgabe des Preises setzte sich auch international fort in den weiteren Reaktionen auf diese Wahl des Komitees. Die österreichische Journalistin und Schriftstellerin Eva Menasse z.B. kommt nach ihrer Lobrede auf Alice Munro in der Zeit zu der Schlussfolgerung: "Dass Alice Munro den Literaturnobelpreis 2013 bekommt, ist eine Nachricht, die mehr Schriftsteller auf der ganzen Welt mit tiefer, ehrlicher Freude und Zustimmung erfüllt als meistens in den Jahren zuvor” (Menasse 2013).

Was ist das Besondere an dieser spezifischen Wahl, die praktisch ausschließlich zustimmende, ja begeisterte Reaktionen hervorgerufen hat, die zu geradezu gerührten und emotional bewegenden Lobpreisungen dieser Schriftstellerin geführt hat? Es gibt diverse Gründe dafür, die ich zunächst in fünf Punkten zusammenfassen möchte.

Zum einen geht diese höchste literarische Auszeichnung damit zum ersten Mal in über hundert Jahren der Existenz dieses Preises nach Kanada, was mehr als überfällig war und nicht nur in Kanada Begeisterung hervorgerufen hat,

\footnotetext{
*Corresponding author: Prof. Dr. Reingard M. Nischik, Chair of American Literature, Department of Literature, University of Konstanz, Fach 166, 78457 Konstanz, Germany, e-mail: reingard.nischik@uni-konstanz.de
} 
sondern bei Kanadisten weltweit. Dass ein Land mit international anerkannten literarischen Koryphäen wie Margaret Atwood, Alice Munro und Michael Ondaatje bis dahin noch nie den Literaturnobelpreis erhalten hatte, war zunehmend unverständlich. Nun hingegen betonte ein begeisterter Douglas Gibson, Munros langjähriger kanadischer Verleger, in einem postkolonialen Land, das erst seit den 1960er Jahren kulturell zu sich selbst fand und seitdem eine kulturelle Blütezeit erlebte: "All of Canada is just delighted by this news [...] - it's as if all of Canada has won the award." "It's a great day for all of us, every Canadian should be walking a little taller” (CBC News 2013 and CTV News 2013).

Ein zweiter Grund für die große Zustimmung zu dieser Preiswahl ist, dass Alice Munro 2013 aus insgesamt 110 Literaturpreisvergaben erst die 13. Schriftstellerin war (und ist), die diesen begehrten Preis erhielt. Da kreatives Schreiben kein Gebiet ist, über das sich behaupten ließe, dass es männliche Schriftsteller neunmal so gut oder überhaupt besser beherrschten als weibliche Schriftsteller, war (und ist) die dekadenlange Hintanstellung weiblicher Schriftsteller mit einem Geschlechterverhältnis von etwa 1:9 bei der Preisvergabe gelinde gesagt sachfremd. Dass in den letzten 23 Jahren nun sieben, also etwa ein Drittel der Preise an Schriftstellerinnen ging (nachdem es in 90 Jahren zuvor nur sechs Preisträgerinnen gegeben hatte), ist immerhin ein Anzeichen dafür, dass das Komitee mittlerweile, gesunderweise, eher mit beiden, nicht nur mit einem Auge Nominierungen liest und bewertet.

Aus literaturwissenschaftlicher Sicht noch bemerkenswerter ist drittens, dass mit Alice Munro eine Schriftstellerin ausgezeichnet wurde, die i.W. Short Stories verfasst hat. Das hatte es bislang noch nicht gegeben und stellt international eine gewichtige symbolische Aufwertung der Gattung der Kurzgeschichte dar, die ja lange im Schatten des Romans stand - nicht so allerdings in Kanada: Die Short Story ist wiederholt als die herausragende Literaturgattung Kanadas bezeichnet worden (s. diverse zitierte Aussagen in Nischik 1987, sowie Nischik 2007a), und bereits zu Beginn der 1980er Jahre war Munros Short Story Band The Moons of Jupiter (1982) derjenige Prosaband (die Romanform also eingeschlossen), für den Penguin den bis dato höchsten Preis für Taschenbuch-Verkaufsrechte kanadischer Literatur zahlte.

Weniger relevant, aber doch auch bemerkenswert an dieser Preisvergabe ist viertens, dass der Preis an eine Schriftstellerin ging, die für ihren zurückgezogenen Lebensstil bekannt ist, die sich - außer mit ihrem literarischen Werk - nicht in öffentliche Diskurse, etwa politisch, einbringt, wie z.B. ihre seit Jahrzehnten im Rampenlicht stehende kanadische Schriftstellerkollegin und Freundin Margaret Atwood; Munro hingegen scheint über sechs Dekaden hinweg neben ihrer Familie nur am Schreiben als solchem, nicht oder kaum an erfolgsbedingter publicity, interessiert gewesen zu sein. Munro wirkt geradezu öffentlichkeitsscheu, 
öffentliche Auftritte oder Lesungen von ihr haben Seltenheitswert - und so passte es auch ins Bild, dass sie den Nobelpreis nicht persönlich in Stockholm entgegen nahm (sondern sich von einer ihrer Töchter vertreten ließ). Wie Munro einmal in den 1980er Jahren sagte, die beiden Hauptinteressen ihres Lebens zusammenführend: "All I want is find time to write - what with my husband and my children - well, they're grown up now [...] but still.”" Eine aus heutiger Sicht komische, wenn auch im Grunde diskriminierende Variante dieser Aussage fand sich 1961 als Titel eines Berichts über Munro in der Vancouver Sun: "Housewife Finds Time to Write Short Stories."

Man sollte den häuslichen Wirkungskontext jedoch nicht geringschätzen, denn er bietet für schriftstellerische Tätigkeit nicht die schlechtesten Voraussetzungen - wie man paradigmatisch an Munros Lebenswerk sehen kann. Damit sind wir bei der fünften und letztlich wichtigsten Besonderheit dieser Auszeichnung, die bei Literaturnobelpreisvergaben nicht immer selbstverständlich war: Alice Munro ist eine grandiose Schriftstellerin, die ihr Metier virtuos beherrscht (wie ich im letzten Teil dieses Artikels demonstrieren möchte) und in ihrer Schreibweise gerade auch für viele Schriftsteller vorbildlich ist - nicht umsonst wird sie auch immer wieder ein "writer's writer" genannt ${ }^{2}-$ was in ihrem Fall allerdings den Erfolg beim allgemeinen Lesepublikum keineswegs ausschloss: Munro ist bereits seit Jahrzehnten Bestseller-Autorin in Kanada, den USA und Großbritannien gewesen, aber auch in anderen Ländern wie Deutschland hatte sie in Übersetzung bereits vor dem Nobelpreis eine angestammte Leserschaft. ${ }^{3}$ Nicht nur ihre Verkaufserfolge und ihre Resonanz in der internationalen Literaturwissenschaft, sondern auch Munros literarische Preise wiesen zunehmend nach oben: Sie erhielt bereits für ihren allerersten Short Story Band 1968 den angesehensten Literaturpreis Kanadas, den Governor General's Award for Fiction - zwei weitere Preise dieser Kategorie sollten folgen (1978 für Who Do You Think You Are? und 1986 für The Progress of Love). Sie erhielt u.a. zweimal den in Kanada höchstdotierten Giller Preis (1998 für

1 Aus einem Interview mit Munro, Quelle nicht eruierbar.

2 S. hierzu Resnick (2012, 122): "A writer's writer [...] is a writer that other writers read, and admire, and study, and try to learn from. And sometimes fall a little bit in love with."

3 Zum Vergleich: Zu viel Glück (Too Much Happiness) belegte im Hardcover-Bereich Rang 29 am 2.10.2010, nach dem Nobelpreis im Taschenbuch-Bereich Platz 1 am 28.10.2013. In einer Rezension vom 4.12.1981 zu Das Bettlermädchen in der Zeit wird Munro als eine "bei uns noch unbekannte kanadische Autorin" bezeichnet (wobei sich dies damals nur auf die allgemeine Leserschaft beziehen ließ, denn in kanadistischen Fachkreisen war Munro auch damals bereits hierzulande wohlbekannt und sehr geschätzt). In einer FAZ Rezension von Die Liebe einer Frau vom 2.12.2000 wird Munro dann als eine auch hierzulande "renommierte Autorin" tituliert. 
The Love of a Good Woman und 2004 für Runaway), dreimal den Ontario Trillium Award (1990, 1999 und 2013), zweimal den Commonwealth Writers Prize (1991 und 2004) sowie den amerikanischen National Book Critics Circle Award (1998 für The Love of a Good Woman). Und als Munro schließlich 2009 auch den renommierten Man Booker International Prize für ihr Lebenswerk ${ }^{4}$ bekam, dachte man spätestens dann, dass dies vermutlich noch nicht die allerhöchste Stufe der Preisverleihungen an sie war.

\section{Kurzbiographie: Vom kanadischen Kleinstadtleben zur weltliterarischen Berühmtheit}

Wer ist dieses Jahrhunderttalent, das Kanada wie auch der Short Story den ersten Literaturnobelpreis bescherte? Eine Grundkenntnis der Biographie Munros zeigt zahlreiche Parallelen zwischen Leben und Werk. Alice Laidlaw wurde 1931 auf einer Farm am Rande der Kleinstadt Wingham, Ontario, geboren, im sogenannten Huron County, dem ländlichen Gebiet, das östlich an den Lake Huron in Kanada angrenzt. Alice war die älteste dreier Kinder einer ehemaligen Lehrerin und eines Farmers - das Aufwachsen auf solch einer Farm in einem traditionellen Geschlechterrollenkontext verarbeitet Munro z.B. in ihrer frühen Erzählung "Boys and Girls” von 1964 (s. Nischik 2007b). Während ihres Englisch-Studiums an der University of Western Ontario (heute: Western University) in London, Ontario, lernte sie ihren Kommilitonen James Munro kennen, den die 20-jährige Alice 1951 heiratete, nachdem sie ihr Studium nach zwei Jahren aus Geldmangel abgebrochen hatte. Das Paar zog an die Westküste Kanadas, zunächst nach Vancouver und in den 1960er Jahren noch weiter westlich nach Victoria auf Vancouver Island. In Victoria gründeten sie ein Buchgeschäft, Munro’s Books (das noch heute existiert und immer noch von Jim Munro geleitet wird). Alice Munros Stories mit Settings an der Westküste Kanadas gehen auf diese Jahre zurück, in denen Munro auch drei Töchter gebar, ein familiärer Hintergrund, der sich auch in ihren Stories niederschlägt. Alice' eigene Mutter erkrankte an Parkinson, als Alice erst 11 Jahre alt war und

4 Indirekt bezeichnend ist, dass Munro weniger häufig auch speziell gattungsbezogene Preise wie den PEN Malamud Award for Excellence in Short Fiction oder den Canadian Authors Association's Jubilee Award for Short Stories erhielt (1997). 
verstarb, als Alice 28 war, eine einschneidende Erfahrung, die sie im gleichen Sommer in "The Peace of Utrecht" (Munro 1960, Dance) und später in "The Ottawa Valley" (Munro 1974, Something) und "Friend of My Youth" (Munro 1990, Friend) literarisch verarbeitete. Nachdem ihre erste Tochter geboren wurde, verkaufte Alice Munro 22-jährig ihre erste Short Story 1953 an eine kanadische Zeitschrift und wurde sodann einem nationalen Publikum auch durch Robert Weavers CBC Anthology bekannt, einer langlebigen kanadischen Radiosendung, in der Short Stories z.T. auch durch die AutorInnen selbervorgelesen wurden. Nach der Geburt ihrer zweiten Tochter schrieb Munro regelmäßig Short Stories und publizierte sie zunächst nur in kanadischen Zeitschriften wie Tamarack Review oder Chatelaine. Nach siebzehn derartig erstpublizierten Short Stories schaffte Munro 1968 ihren nationalen Durchbruch gleich mit ihrer ersten, preisgekrönten Short Story Sammlung Dance of the Happy Shades. Und ihr zweiter Short Story Band, als "Roman” vermarktet, Lives of Girls and Women (1971), bereitete ihren endgültigen internationalen Durchbruch in den 1970er Jahren mit Who Do You Think You Are? (1978) vor. 1973, nach 22 Jahren, scheiterte ihre erste Ehe mit Jim Munro und nach zwei Jahrzehnten in British Columbia an der kanadischen Westküste zog es sie zurück in ihre Heimat nach Ontario. Dort ließ sich Alice ab 1975 mit ihrem zweiten Ehemann, dem im April 2013 verstorbenen Gerald Fremlin, in dessen Elternhaus in Clinton, Ontario, nieder. Dort, in Clinton, zurück in ihrem geliebten südwestlichen Ontario, nur $30 \mathrm{~km}$ von ihrer Geburtsstadt Wingham entfernt, hat Munro die nächsten 40 Jahre bis heute gelebt. Sie hat kurioserweise meistens in ihrer Schriftstellerkarriere kein eigenes Arbeitszimmer gehabt - eine Erfahrung, die sie in "The Office" (Munro 1962, Dance) literarisch verarbeitete - in ihrem Haus in Clinton hatte ihr Ehemann, ein Geograph, ein Arbeitszimmer, sie hingegen arbeitete an einem kleinen Schreibtisch im Esszimmer, mit Blick auf die Hauszufahrt. Es scheint ihr nicht geschadet zu haben: So begann 1976 ihre jahrzehntelange Verbindung mit der amerikanischen Monatszeitschrift The New Yorker, vermutlich die weltweit "erste Adresse" für Erstveröffentlichungen von Short Stories - und bezeichnenderweise sicherte sich diese Zeitschrift, die Munros Werk speziell auch in den USA bekannt machte, das Erstzugriffsrecht auf ihre Short Stories - kein anderer Autor, keine andere Autorin hat so lange und so viele Short Stories im New Yorker erstpubliziert wie Alice Munro. ${ }^{5}$

5 Zum potentiellen Einfluss des New Yorker auf Munros Werk s. Beran (1999). S. auch Munros "Author's Note" zu Beginn von The Love of a Good Woman: "Stories in this collection that were previously published in The New Yorker appeared there in very different form” (Munro 1998). 


\section{Alice Munros Kunst der Short Story: Gesamtwerk, Charakteristika, Poetologie}

Das Gesamtwerk der heute 83-Jährigen erstreckt sich über ein halbes Jahrhundert und umfasst insgesamt 14 Short Story Sammlungen, publiziert zwischen 1968 und 2012.

Der jüngste Band mit dem resonanten Titel Dear Life (2012; deutsch: Liebes Leben) wird nach eigener Aussage Munros ihr letzter sein, auch da sie die Energie, die das Schreiben erfordert, nicht mehr aufbringen kann. Ein Bericht über die (bereits zweite) Rücktrittserklärung Munros vom Schreiben publiziert im Juli 2013 in der New York Times ist betitelt "Alice Munro Puts Down Her Pen to Let the World In," und sie wird darin zitiert mit den Worten:

There will be no more books after Dear Life [...] I don't have the energy anymore [...] it's very hard, and you get very tired [...] I feel a bit tired now - pleasantly tired [...] I feel that I've done what I wanted to do, and that makes me feel fairly content. (McGrath 2013, 1-3)

So sieht es also danach aus, als würden wir nun, nach der Nobelpreisvergabe an Munro, ein abgeschlossenes Gesamtwerk überschauen.

Was sind die Charakteristika der Short Stories Munros, die ja z.T. schon anklangen, was ihre poetologischen Bedingungen und Finessen? Zum einen zählt Munro zu den SchriftstellerInnen, die eine bestimmte Region und ihre Bewohner auf die weltliterarische Landkarte setzten und damit sozusagen verewigten: die Rede ist von Südwest-Ontario, speziell das Huron County um London, Ontario, herum, heute manchmal auch "Munro Country" genannt. Von ihren frühen Texten (z.B. "Walker Brothers Cowboy” 1968) bis zu ihrem Spätwerk (z.B. "Dear Life” 2011) hat sie diese Region ihres Lebens immer wieder literarisch aufgerufen und rekreiert. Obwohl diverse ihrer Stories auch andere, z.T. internationale Settings haben, wie das westliche Kanada oder z.B. Schottland (wo die familiären Wurzeln ihrer Vorfahren liegen), Australien oder Albanien, sind doch die meisten ihrer Stories im ländlichen und kleinstädtischen Kontext des südwestlichen Ontarios angesiedelt. Wie so häufig bei SchriftstellerInnen verweigert sich Munro aber allgemeinen Zugehörigkeitszuschreibungen und relativiert ihre häufige Klassifizierung als Regionalschriftstellerin so:

A lot of people think I'm a regional writer. And I use the region where I grew up a lot. But I don't have any idea of writing to show the kind of things that happen in a certain place. These things happen and the place is part of it. But in a way, it's incidental. (Munro in Hancock 1987, 200) 
Mit anderen Worten, Munro hat letztlich kein dokumentarisches Interesse bei der Wahl ihres bevorzugten Settings, obwohl das Beschreiben regionaler Besonderheiten durchaus ein Effekt ihres Schreibens ist; sie ist vielmehr an übergreifenderen Themen menschlicher Befindlichkeit interessiert, die über die dargestellte Region hinausweisen. Zudem rekreiert sie in Bezug auf Settings weniger das Bekannte oder Wiedererkennbare, sondern fokussiert in ihrem Ergründungsversuch dessen, was sie einmal metaphorisch "deep caves paved with kitchen linoleum” (Munro 1971b) nannte, auf das Rätselhafte, Unsichtbare, Überraschende, Verfremdete der ihr bekannten Region. In dieser Ausrichtung sieht man auch den Einfluss von Schriftstellerinnen des amerikanischen Südens wie Flannery O’Connor, Carson McCullers und besonders Eudora Welty auf das Werk Munros, sodass man in Zusammenhang mit Munros Texten häufiger auch von "Ontario Gothic” spricht (s. Berndt 2010). Wie Munro selber einmal über Südwest-Ontario sagte: "The part of the country I come from is absolutely Gothic. You can't get it all down” (Munro in Gibson 1973, 248).

Neben der von Munro bevorzugten regionalen Verortung, die z.B. auch realitätskonforme Benennungen von Orts- und Straßennamen einschließt, haben ihre Short Stories als zweites Charakteristikum meistens weibliche Protagonisten, werden aus weiblicher Wahrnehmungsperspektive geschrieben und umfassen entsprechende thematische Akzentuierungen: Mutter-Tochter Bezüge, Tochter-Vater Bezüge, Schwester-Bruder Bezüge; restriktive Geschlechterrollen, geschlechtsbezogene berufliche Problematiken, oft auch im künstlerischen bzw. schriftstellerischen Kontext; Liebesbezüge und Partnerschaftsprobleme, das Wunder und Wunderbare der Liebe wie auch ihr Scheitern bzw. ihre Vergänglichkeit; oder Probleme des Älterwerdens und schließlich Alterns - all dies also i.W. aus einer weiblichen Wahrnehmungsperspektive dargestellt, die auch auf Munros eigenes Leben zurückweist.

In der Tat, die regionalen Ursprünge und genderspezifische Akzentuierung ihrer Thematiken weisen auf das dritte Hauptcharakteristikum ihres Schreibens, die starken autobiographischen oder, wie Munro selber sagt, "persönlichen" Wurzeln und Dimensionen ihres Werkes (Munro in Struthers 1981, 17). Es lässt sich argumentieren, dass Munro tendenziell entlang ihrer eigenen Lebenserfahrungen schreibt, zu Beginn ihrer Karriere fokussierend auf die Aufarbeitung von Kindheit und Heranwachsen, über Berufs- und Partnerschaftserfahrungen, bis hin zum Alterungsprozess - und so werden nicht nur die Stories von Munro im Laufe der Jahrzehnte tendenziell länger, sondern auch ihre HauptCharaktere entsprechend älter ${ }^{6}$ - bis Munro nun, folgerichtig, angekündigt

6 So auch Howells: "with an accompanying sense of individual lives scrolling out over many decades” (Howells 2003, 54). 
hat, fortan literarisch zu verstummen: "I've done what I wanted to do, and that makes me feel fairly content” (s. McGrath 2013). In gewisser Weise lässt sich Munros Gesamtwerk also als eine große, diskontinuierliche und variantenreiche Parallelgeschichte zu ihrer eigenen Erfahrungswelt ansehen - nicht umsonst z.B. ist ihre letzte Erzählung “Dear Life” im gleichnamigen letzten Erzählband auch eine bewegende Reminiszenz an ihre Mutter und ist ein sehr kompliziertes, unabgeschlossenes Mutter-Tochter-Verhältnis ein wesentliches Motiv, das sich in vielen Erzählungen Munros findet. Hier ist die bewegende Schlusspassage der Erzählung “Dear Life,” möglicherweise nicht nur das Endes dieses Erzählbandes, sondern gemäß ihrer eigenen Aussage auch der Schlussakkord ihrer gesamten Schriftstellerkarriere:

I did not go home for my mother's last illness or for her funeral. I had two small children and nobody in Vancouver to leave them with. We could barely have afforded the trip, and my husband had a contempt for formal behavior, but why blame it on him? I felt the same. We say of some things that they can't be forgiven, or that we will never forgive ourselves. But we do - we do it all the time. (Munro 2012, 319)

Was viele Fachleute und LeserInnen besonders an Munros Texten fasziniert - und das ist das vierte und vielleicht wichtigste Hauptcharakteristikum ihres Werkes ist weniger das Was als das Wie ihrer Erzählungen, weniger der Erzählinhalt als die Erzählform oder, besser, der Erzählinhalt in Kombination mit der Erzählform: Sprachstil und Erzähltechnik, die Art der erzählerischen Vermittlung. Gerade bei Munros Texten greifen Hauptaussagen und Erzählform in elaborierter Weise ineinander, bedingen sich, was auf einer komplexen Rezeptionsebene nichts weniger als Staunen ob solch einer raffinierten und dennoch verständlichen, ja sehr lesbaren Erzählkunst hervorruft. Munro weitet die Grenzen der Gattung der Short Story, experimentiert mit der Gattungsform in ihrem non-linearen, flächigen, scheinbar digressivem, oftmals montageartigem Erzählstil (z.B. "Dulse" in Moons), schreibt in multiperspektivischer, epiphanischer Erzählweise (z.B. "Lichen" in Progress), fördert das Außerordentliche, Wunderbare, aber auch Bedrohliche im Alltäglichen zutage. Häufig denken ihre Texte auch in selbstreflexiven Passagen über das kreative Schreiben an sich, das Verhältnis von Realität und Fiktion nach, integrieren also poetologische Problematiken in die Erzählungen: "And what happened, I asked myself, to Marion? [...] Such questions persist, in spite of novels. It is a shock, when you have dealt so cunningly, powerfully, with reality, to come back and find it still there" (Munro 1971b, 247).

In derartigen Passagen betont Munro immer wieder das Fragmentarische, Unabgeschlossene und Unergründbare menschlicher Erfahrung sowie die letztendliche Unfähigkeit literarischer Texte, den weiten Fluss auch individuellen Lebens literarisch-definitiv in den Griff $\mathrm{zu}$ bekommen. Es kann immer nur 
Annäherungs- und Erklärungsversuche geben, und wessen man sich noch am sichersten sein kann ist der jeweilige Moment, der vorübergehende, auch schon nur annäherungsweise "still point of the turning world," wie T.S. Eliot es einmal formulierte. Munros Ästhetik ist insofern eine Ästhetik des Augenblicks, ihre Texte eine Reihung von Episoden, zwischen denen häufig eher die Verbindungslücken denn die Erklärungsbrücken deutlich werden. Das Ergebnis einer solchen porösen Sichtweise von menschlicher Erfahrung und Literatur sind Texte, die Erklärungslücken ausstellen, die unterschiedliche Interpretationen und verschiedene Sichtweisen der gleichen Situation, auch des gleichen Charakters, oft zeitlich versetzt, gegeneinanderstellen. Es finden sich Gegenübersetzungen von Vergangenheit und Gegenwart, die durch das Montageartige der Darstellung eher ein Nebeneinander denn eine Entwicklungslinie suggerieren, "worlds alongside," also 'Welten nebeneinander,' wie es Munro in Lives of Girls and Women einmal formulierte. ${ }^{7}$ Das Strukturprinzip der parallelen Ergänzung, Verbreiterung wird also gegenüber der linearen Abfolge aufgewertet. Es ereignen sich fast unmerkliche Perspektivenwechsel und ein ständiges Verschieben, ja Aufschieben von etwaigen fixen Bedeutungen, was immer wieder die Fluidität, Veränderlichkeit, Uneindeutigkeit und letztliche Unergründbarkeit menschlicher Existenz unterstreicht. Wie es in Munros Story “The Ottawa Valley” (in Something 1974) mit metafiktionaler Aussagekraft heißt: "I wanted to find out more, remember more. I wanted to bring back all I could. Now I look at what I have done and it is like a series of snapshots" (Munro 1974b, 197).

So fügen sich Munros literarische Ansichten in der Tat am kongenialsten in das Format der Short Story, die ausschnittsartige Sichtweisen des Lebensflusses, episodische und kondensierte Zeitstruktur, fragmentarische Darstellungsformen und offenes Ende privilegiert. Auch der von Munro bekannten Sprachstilversessenheit und ihrem Hang zur vielfachen, langwierigen Überarbeitung ihrer Texte kommt die kürzere Prosaform entgegen. Selbst in den früheren beiden Büchern, in welchen Munro sich an der Romanform versuchte, i.W. auf Drängen ihres damaligen Verlegers, ergibt sich immer wieder die Short Story als Aufbauzelle, trotz verzweifelten Integrationsbemühungen durch die Autorin sogar noch im Fahnenkorrekturstadium ${ }^{8}$ - herauskam, was ich Kurzgeschichtenzyklen, nicht Romane nennen würde (nämlich Lives und Who). Wie auch Munro selber dann in den 1980er Jahren betonte: "I think the most attractive kind of writing of all is just the single short story. It satisfies me the way nothing else does [...] It took me

7 "So lying alongside our world was Uncle Benny's world like a troubling distorted reflection, the same but never at all the same” (Munro 1971a, "The Flats Road” in Lives, 26).

8 S. hierzu Munro selber auf die Frage eines Interviewers zu ihrem Überarbeitungsprozess von Who: “Oh, it's the most confused revision in history” (Struthers 1981, 29 passim). 
a long time to reconcile myself to being a short-story writer" (Munro in Hancock 1987, 190). Und obwohl ihre Stories in ihren späteren Sammlungen tendenziell länger werden, ist Munro bis zum Schluss ihrer bevorzugten Erzählgattung treu geblieben und weitet sie gemäß ihrer literarischen Bedürfnisse.

Das was Coral Ann Howells die 'Unbestimmtheit' (“indeterminacy," Howells 1990) in den Erzählungen Munros nennt, die sie virtuos für ihre Zweckeeinzusetzen vermag, beeinflusst sowohl Munros Erzähl- wie auch ihren Sprachstil (besonders ab The Moons of Jupiter 1982) und fügt sich zu resonanzreichen, leseraktivierenden literarischen Kompositionen. Man betrachte nur einmal die Aussagekraft dieses einen Satzes aus Lives of Girls and Women (Munro 1971b, 249): “People’s lives, in Jubilee as elsewhere, were dull, simple, amazing, and unfathomable - deep caves paved with kitchen linoleum." Nur schon in diesem einen Satz finden wir eine über das Lokale bzw. Regionale hinausweisende, generalisierende Bedeutungsausweitung, sowie gleichzeitig eine hier oxymoronisch angelegte, also sich in sich widersprechende Unbestimmtheit: wenn Leben "dull”/'langweilig,' 'stumpf' sind, können sie eigentlich schwerlich gleichzeitig "amazing”/'erstaunlich,' 'verblüffend,' 'wunderbar' sein; wenn sie “simple”/'einfach' sind, können sie gleichzeitig kaum "unfathomable”/'unergründbar' sein - und doch sind sie in der in Lives dargestellten Wahrnehmungsperspektive der heranwachsenden Schriftstellerin all dies gleichzeitig, werden sie vielperspektivisch interessant durch den hinter die Fassade des Alltäglichen, des Küchenlinoleum, schauenden, um mehrdimensionale Wahrnehmung bemühten Schriftstellerblick, den die “deep caves,” die ‘tiefen Höhlen' des Menschlichen hinter der Alltagsfassade herausfordern. Insofern hat auch dieser Satz metafiktionale Aussagekraft. Das Unbestimmtheitsprinzip und sein bedeutungsmäßiger Mehrwert, der sich hier schon an einem einzigen, zudem recht kurzen Satz der früheren Schaffensperiode Munros zeigt, weitet besonders ihre mittleren und späteren Erzählungen. Die Texte etablieren Resonanzräume, in denen verschiedene Bedeutungs- und Deutungsmöglichkeiten zirkulieren und sich eindeutigen, definitiven Plotentwicklungen und Interpretationen entziehen. Durch die oben skizzierten Erzähltechniken wird eine eindeutige Erzähllinie immer wieder durchbrochen. Das Ergebnis sind mehrdimensionale Erzählungen, die Intellekt und Emotion gleichermaßen ansprechen und in ihrer auch affektiven Wirkung auf die entschlüsselungsangereizte Leserin noch lange nachhallen. Was Munro selber am Ende ihrer Story “Simon's Luck” in erzählerischem Kontext formuliert, lässt sich so auch, wiederum selbstreflexiv, auf ihre eigene, nun nobelpreisgekrönte Erzählweise beziehen. Die Protagonistin Rose sinniert am Ende der Story als sie, quälerisch verspätet, vom Tod ihres neuen, von ihr so stark begehrten Geliebten Simon erfährt, über "those shifts of emphasis that throw the story line open to question, the disarrangements which demand new judgments and solutions, and 
throw the windows open on [...] unforgettable scenery" (Munro 1978 Who Do You Think You Are? / The Beggar Maid, 177).

\section{Beispielanalyse: Alice Munro, “Fiction” (2007)}

Von den wahrlich zahlreichen Stories, die hier beispielhaft analysiert werden könnten - Munro hat in ihren 14 Sammlungen insgesamt 147 Short Stories publiziert - habe ich mich für eine spätere Erzählung entschieden, nämlich "Fiction" (dt. "Erzählungen") aus Too Much Happiness (2007). Die Story umfasst, bis auf das von Munro bevorzugte Setting des südwestlichen Ontario, alle der oben skizzierten Charakteristika ihrer Schreibkunst.

Der 29 Seiten umfassende Text ist in zwei Sektionen unterteilt, jeweils mit vorangestellten römischen Zahlen I und II voneinander abgesetzt. Teil I ist in einem Waldgebiet hinter der Ortsgrenze des fiktionalen Rough River an der kanadischen Westküste angesiedelt. In dem Waldgebiet, in dem alle 400 Meter ein Haus oder Wohnwagen steht mit Bewohnern, die einen naturbezogenen, alternativen Lebensstil pflegen, haben sich die Protagonistin Joyce und ihr Ehemann Jon in einem einfachen, selbstrenovierten Haus niedergelassen. Joyce ist Musiklehrerin und kommt von ihrem Arbeitstag an den Rough River Schools zurück. Sie freut sich auf das Heimkommen, auch auf ihren Mann Jon, der passionierter Schreiner ist und zuhause arbeitet.

Der Beginn der Erzählung evoziert das landschaftliche Setting an der kanadischen Westküste und betont bereits im zweiten Satz, scheinbar beiläufig, wie nahe wesentliche Unterschiede im Alltag beieinander liegen können:

The best thing in winter was driving home, after her day teaching music in the Rough River schools. It would already be dark, and on the upper streets of the town snow might be falling, while rain lashed the car on the coastal highway. (Munro 2007, 32)

Der zweite Absatz fokussiert sodann auf Joyce' Freude am abendlichen Heimkommen und ihre Vorliebe für die erleuchteten Terrassentüren an den Häusern, die mit ihren Einblicksvermittlungen zum einen behagliche Sicherheit und Gemütlichkeit des Zusammenwohnens drinnen ausstrahlen: "These [patio doors] were usually left uncurtained, and [...] seemed to be a sign or pledge of comfort, of safety and replenishment [...] they displayed the haven of home so artlessly" (Munro 2007, 32-33). Dieser (scheinbaren) häuslichen Behaglichkeit und Sicherheit, die Joyce, wie an der Passage deutlich wird, sehr wichtig ist, werden im gleichen kurzen Absatz allerdings auch zwei gegenläufige Aspekte entgegengesetzt (Supplementaritätsprinzip): Zum einen ist Joyce bewusst - in 
einer weiteren Variation des Munroschen Fassadenmotivs - dass das, was von außen betrachtet behaglich-attraktiv erscheint, aus einer Innen-Sicht ganz anders erscheinen mag ("scenes that beguiled her, even if she knew things would not be so special inside”; Munro 2007, 33) - auf die Perspektive kommt es an. Zum zweiten wird durch ihr Hineinschauen in beleuchtete Häuser durch sicht-ungeschützte Terrassentüren gleich zu Beginn das sich bald als zentral erweisende Motiv des Eindringens bzw. Eindringlings in private Behaglichkeit evoziert.

Darauf folgt eine Art Exposition, mit stark raffenden zeitlichen Rückwendungen zu Joyce' und Jons Schulzeit, als sie sich kennenlernten, und wie sie nach ihrem ersten College-Jahr das Studium abbrachen und auf Reisen gingen, in einer Art Hippiedasein den nordamerikanischen Kontinent bereisten und sich schließlich in den USA an der Oregon-Küste für ein Jahr niederließen, bevor sie sich zu einer Ausbildung und einem etwas gesetzteren Lebensstil entschlossen und so in der Nähe von Rough River an der kanadischen Westküste landeten. Auf Betreiben von Joyce ("she believed they had an obligation to society"; Munro 2007, 35) stellt Jon im Rahmen eines Regierungsförderungsprogramms einen weiblichen Lehrling, Edie, an. Was folgt ist wiederum große Erzählkunst: Anhand weniger Unterhaltungen zwischen dem Ehepaar über Edie sowie zwischen Edie und dem Ehepaar wird Edie andeutungsweise als selbstbezogene, selbstbewusste, in ihrer Kommunikation direkte, aber auch apodiktische sowie körperlich starke - und insofern für den Schreiner-Job geeignete - Frau charakterisiert, sodann auch die zunächst verharmlosende, distanzierende Haltung des Ehepaars ihr gegenüber gezeigt: "They would laugh about something Edie had said. But not in a disparaging way - Edie was like a pet, Joyce sometimes thought. Or like a child" (Munro 2007, 35). Auch diese Edie verharmlosende Ansicht, vor allem von Joyce, soll sich bald als Täuschung bzw. erzählerische Ironie erweisen: In stark zusammenraffender Erzählweise wird anschließend auf ca. einer Seite gezeigt, dass a) Jon sich in Edie verliebt und b) die Beziehung des Ehepaars deswegen beendet ist. Der zunächst kaum merkliche Umschwung ergibt sich aus Jons Erzählung, dass Edie, eine ehemalige, aber nun trockene Alkoholikerin, offene Weinflaschen auf dem Küchentisch des Ehepaars schwer ertragen könne. Es folgt die Kommunikation zwischen Jon und Joyce, die die umwälzende Veränderung in ihrer aller Leben markiert, zunächst indirekt, und dann - offenkundig aus der Retrospektive erzählt ${ }^{9}$ - epiphanisch auf den Punkt bringend:

Also, she thought Jon and Joyce - well, really Joyce - should not leave wine bottles with wine in them right out in sight on the kitchen table.

9 Teil I wird in der Vergangenheitsform, Teil II in der Gegenwartsform erzählt. 
“That's her business?” said Joyce.

"Apparently she thinks so."

"When does she get to examine our kitchen table?"

"She has to go through to the toilet. She can't be expected to piss in the bush."

"I really don't see what business -"

"And sometimes she comes in and makes a couple of sandwiches for us-"

"So? It's my kitchen. Ours."

"It's just that she feels so threatened by the booze. She's still pretty fragile. It's a thing you and I can't understand.”

Threatened. Booze. Fragile.

What words were these for Jon to use?

She should have understood, and at that moment, even if he himself was nowhere close to knowing. He was falling in love. (Munro 2007, 37)

Joyce sinnt sodann über die Implikationen der wörtlichen Bedeutung dieses speziellen, für sie völlig unverständlichen "falling in love" nach, das aus ihrer Sicht im vorliegenden Fall entsprechend negativ ausgedeutet wird: "No way this could be seen as probable or possible, unless you think of a blow between the eyes, a sudden calamity. The stroke of fate that leaves a man a cripple, the wicked joke that turns clear eyes into blind stones" (Munro 2007, 37-38). Der anschließende, zunächst noch optimistisch angesetzte Rettungsversuch der Ehe durch Joyce (“We will ride this out,' she said”), scheitert auf kleinstem erzählerischen Raum - mit nur vier von Jon ausgesprochenen Worten: "There is no 'we,' he said" (Munro 2007, 38).

Darauf folgen einige Szenen, die das Unverständnis und vor allem die Verzweiflung von Joyce zeigen: "Her life gone. A commonplace calamity" (Munro 2007, 40). Sie zieht aus dem gemeinsamen Haus aus und nimmt sich eine Stadtwohnung, vergeblich hoffend, dass Jon zu ihr zurückkehrt - stattdessen zieht Edie mit ihrer Tochter Christine zu Jon in das Haus. Joyce engagiert sich nun besonders stark in ihrem Beruf der Musiklehrerin und versucht auch darüber hinaus, allen, aber besonders Männern, zu gefallen - all ihr Verhalten ist jedoch, wie aus Joyce' Fokalisierungsperspektive klar gemacht wird, auf Jon ausgerichtet, den sie auf diese Weise, da ihr nichts anderes mehr zu bleiben scheint, zu beeindrucken versucht:

She had some idea that Jon would hear about how pretty she looked, how sexy and happy, how she was simply bowling over all the men. As soon as she went out of the apartment she was on stage, and Jon was the essential, if second-hand, spectator. (Munro 2007, 40-41)

Doch obwohl Edies Tochter Christine Joyce' Schülerin ist und auch beim dieses Mal von Joyce besonders engagiert vorbereiteten Schuljahr-Abschlusskonzert auftritt - Joyce hofft, dass Jon und Edie zum Konzert kommen - lassen sich Jon und Edie nicht blicken, sondern scheinen den Kontakt zu Joyce zu meiden. 
Der zweite Teil der Story beginnt mit einem abrupten Zeit- und Ortssprung: Wir befinden uns plötzlich auf einer Party von Joyce und Matt, Joyce' zweitem Ehemann, mit dem sie in einem großen Haus in North Vancouver lebt. Joyce ist in der Zwischenzeit Berufsmusikerin geworden, eine - mittlerweile ergraute - Cellistin, ihr 65-jähriger Mann Matt, für den Joyce die dritte Ehefrau ist, ist Neuropsychologie-Professor. Das Leben des kulturell interessierten Paares Joyce und Matt ist ereignisreich und erfüllt, von vielen sozialen, familiären und außerfamiliären, Kontakten geprägt und Matt und Joyce sind erfolgreich in ihren Berufen. Sie scheinen eine zufriedene Ehe zu führen, die Gemeinsamkeit im Bett scheint oft in gemeinsamem Lesen und Austausch darüber zu bestehen. Es wird erwähnt, dass Joyce auch Jon - der ebenfalls ein drittes Mal verheiratet ist, also Edie in zweiter Ehe geheiratet hatte - zu Joyce' und Matts Geburtstagsparty eingeladen hat, aber Jon hat wegen anderer familiärer Verpflichtungen, der Taufe eines Enkelkindes, absagen müssen. Der frühere Lebensabschnitt mit Jon scheint für Joyce weitgehend abgeschlossen, beide haben scheinbar ihr neues Glück gefunden, und es mag Joyce etwas getröstet haben, dass Jons Ehe mit Edie auch nicht von Bestand war.

Und doch greift die Vergangenheit in die wiederum zufrieden, ja glücklich scheinende Gegenwart ein, zunächst noch von Joyce und den LeserInnen unbemerkt. Als Joyce sich auf der Party einer Gruppe mit jüngeren Leuten um ihren Stiefsohn Tommy herum nähert und kurzen, aber geistreichen Smalltalk macht, entfernt sich eine Christie genannte junge Frau aus der Gruppe - wie sie auf Nachfrage sagt, um in den Büschen eine Zigarette zu rauchen. Joyce hat zunächst eine unmittelbare Antipathie der Frau gegenüber, verliert über das diffuse Nachdenken über sie kurz ihre gute Stimmung ("Joyce feels a lot of her cheer drained away"; Munro 2007, 47) und sogar auch etwas ihre Souveränität (“At a loss, for a moment, as to what to do or where to go next"; Munro 2007, 47). Joyce’ Befragung ihres Stiefsohns später beim Abwaschen ergibt, dass diese Frau Christie O'Dell heißt, verheiratet ist und soeben ihre erste Short Story Sammlung veröffentlicht hat. Als Joyce einige Tage später an einem Buchgeschäft vorbei kommt, erkennt sie das Gesicht der Frau auf einem Poster wieder, kauft das Buch und wird von der Verkäuferin über eine Lesung mit der Jungautorin einige Tage später im Buchgeschäft informiert. Zuhause, mit ihrem Mann im Bett lesend, erregt der Titel einer Short Story im gekauften Buch Joyce’ Aufmerksamkeit: “Kindertotenlieder,” der Titel auch eines Liederzyklus von Gustav Mahler. Zuerst neben ihrem lesenden, aber auch mit Joyce kommunizierenden Mann Matt, und dann sich seiner Nähe entziehend in der Küche, verschlingt Joyce die Story “Kindertotenlieder" - und ab hier entwickelt die Munro Story endgültig eine sogartige Intensität: So gefangen wie Joyce die Erzählung Christie O’Dells liest, so fasziniert verfolgt dabei auch die Leserin die allmähliche Enthüllung, dass Christie nur Christine, Edies Tochter 
sein kann, denn in ihrer Short Story schildert Christie O'Dell Dinge, die einer möglichen vergangenen Realität stark ähneln und die so nur sie wissen konnte. Die Story von Christie schildert nämlich aus kindlicher Wahrnehmungsperspektive den Dreiecksbezug einer Musiklehrerin mit ihrem Mann und der Mutter des Kindes, die in die Ehe eindringt und die Musiklehrerin aussticht. Alles was folgt hätte so sein können wie in der Story “Kindertotenlieder” geschildert: Das Mädchen ist vernarrt in die Musiklehrerin, die davon nichts ahnt, die Lehrerin versucht vielmehr Informationen aus dem Mädchen über die Beziehung ihres ExPartners mit der Mutter des Mädchens, mit denen das Mädchen ja nun gemeinsam im ehemaligen Haus der Musiklehrerin lebt, herauszufinden. Joyce ist gebannt von der Lektüre dieser Story. Sie geht einige Tage später ins Buchgeschäft zur Buchsignatur Christie O’Dells, voller Aufregung, weil sie Christies Story scheinbar für bare Münze nimmt, sie also als autobiographische Erzählung auffasst. Sie bringt der Autorin ein Geschenk mit, das aus ihrer Erzählung "Kindertotenlieder" abgeleitet ist, Tulpen aus Schokolade, und versucht festzustellen, ob die Autorin Zeichen des Wiedererkennens Joyce gegenüber zeigt. Das ist nicht der Fall: Christie behandelt Joyce zurückhaltend, so wie auch die anderen LeserInnen, die zur Buchsignatur vor ihr anstehen.

Christie O'Dell sits there and writes her name as if that is all the writing she could be responsible for in this world [...] [She] has raised her eyes to greet the next person in line, and Joyce at last has the sense to move on [...]

Walking up Lonsdale Avenue, ${ }^{10}$ walking uphill, she gradually regains her composure. This might even turn into a funny story that she would tell some day. She wouldn't be surprised. (Munro 2007, 61)

So endet "Fiction," wiederum in selbstreflexiver Manier. Wesentliche Fragen bleiben offen: Warum reagiert Joyce so hochemotional auf die Story "Kindertotenlieder"? "It means a great deal to me. I brought you a present” (Munro 2007, 60), sagt sie zu Christie bei der Buchsignatur. Ist es der bloße Wiedererkennungseffekt? Oder nimmt sie Christies Story für bare Münze und ist geschmeichelt und angerührt von dem Effekt, den sie auf das junge Mädchen damals, gemäß also einer autobiographischen Lesart, gehabt hat? Oder bricht ihre alte Liebe zu Jon wieder auf, die Emotionen und starken Verletzungen ihres damaligen Lebensabschnitts? Und warum verhält sich Christie O’Dell so distanziert Joyce gegenüber? Hat sie sie wiedererkannt oder nicht? Wenn nein, so macht ihre distanzierte Haltung gegenüber einer aus ihrer Sicht dann bloßen Leserin Sinn, aber warum verließ Christie dann sogleich die Gesprächsrunde der jungen Leute, als Joyce bei

10 Eine real existierende Straße in Vancouver. 
ihnen während der Party auftauchte? War Christie überwältigt vom Auftauchen Joyce' oder wollte sie ihr eher entgehen? Wenn Christie Joyce erkennt, was plausibler wäre, so ist ihre Distanziertheit ihr gegenüber verschiedentlich erklärbar: Ihr ist die Offenlegung ihrer damaligen Gefühle Joyce gegenüber, wenn es sich um eine autobiographisch unterlegte Story handelt, vor Joyce unangenehm. Oder - auch eingedenk des Story-Titels "Fiction" - die erwachsene Autorin Christie O’Dell hat diese einseitige Verliebtheitsgeschichte zwischen kindlicher Schülerin und verheirateter Musiklehrerin erfunden, fiktional entwickelt, wenn auch auf der von ihr damals als Kind erlebten persönlichen Grundkonstellation aufbauend. Vermutlich will Munro uns mit dieser ihrer Story kunstvoll, in erzählerischer Manier, vor Augen führen, dass es letztlich, bei aller autobiographischer Inspiration ihrer Erzählungen, doch eben elaborierte Fiktion ist, die sie schreibt, und beileibe keine Lebensbeschreibungen oder -erinnerungen. Gleichzeitig ist "Fiction" aber auch sozusagen ein literarischer Spiegel dafür, wie sehr eigene und fiktionale Erfahrungswelt in Munros Werk ineinandergreifen - denn ohne ihre spezifischen 'Lebenserfahrungen' hätte Christie O’Dell ihre Story “Kindertotenlieder" so nicht geschrieben.

Geht man, in Kenntnis des völlig überraschenden Endes von "Fiction" bezüglich der story within a story "Kindertotenlieder," zum ersten Teil der Munro Story zurück auf der Suche nach etwaigen Hinweisen für eine solche Entwicklungslinie im Bezug zwischen der Musiklehrerin Joyce und ihrer Schülerin Christie, Tochter von Edie, in Joyce' Rough River Zeit, so findet sich dazu sehr wenig, wobei dieser Teil aus der Fokalisationsperspektive von Joyce verfasst ist. Es wird in Teil I nur erwähnt, dass Joyce auch die Tochter Edies unterrichtet und sich insofern besonders anstrengt vor dem Schulkonzert, da sie hofft, dass Jon (mit Edie) im Auditorium sein wird. Es findet sich nur ein, in seiner mehrfachen Bedeutsamkeit beim ersten Lesen zunächst so nicht erkennbarer Hinweis, der die spätere Erzählung Christies sozusagen in der fiktionalen 'Realität' fundieren könnte, allerdings mit völlig anderer Wertigkeit, je nachdem, aus welcher Perspektive ein und dieselbe Situation betrachtet wird. Denn es wird in Teil I in der Tat betont, dass sich Joyce in der Zeit der Vorbereitung des Schul-Konzerts ihren SchülerInnen, sogar auch den jüngsten und unbegabtesten unter ihnen, mit großem persönlichen Engagement zuwendet: "Even with the youngest or the dullest children she taught, her tone had become caressing, full of mischievous laughter, her encouragement irresistible” (Munro 2007, 41). Doch diese Passage wird in Teil I eindeutig in den (ex-)partnerschaftlichen Kontext eingeordnet, dass Joyce alles was sie tut auf Jon ausrichtet, wenn auch letztlich selbstbezogen, hier also an einem erfolgreichen Konzert vor Jon interessiert ist und ihre SchülerInnen deswegen besonders motivieren will (und es wird in Teil I bezeichnenderweise nicht erwähnt, dass Edies Tochter von Joyce die Hauptrolle im Schul-Konzert 
zugesprochen bekommen hätte - so stellt sich das erst in Christies späterer Erzählung dar). Eine derartige Wahrnehmungsdiskrepanz in Bezug auf die objektiv gleiche Situation durch zwei verschiedene Charaktere ist - in Munros Worten - ein solcher oben genannter "shift of emphasis that throws the story line open to question" - die Leserin, die in der erzählerischen Vermittlung auf die Filterung der Geschehnisse durch die Charaktere bzw. die Fokalisationsinstanzen angewiesen ist, fragt sich am Schluss, was denn nun eigentlich die 'wahren' Geschehnisse sind. Die Frage bleibt offen, mit am Textende einem weiter verkomplizierenden Verweis auf eine weitere anstehende Fiktionalisierung (nämlich auf die Story, die wir gerade lesen).

Wir sollten uns also hüten, literarische Texte, auch wenn sie autobiographisch fundiert scheinen, mit der Realität zu verwechseln. Dies zeigt sich auch schön an einer weiteren metafiktionalen Passage der Story "Fiction," in der Munro selbstironisch auf die lange Zeit geringere Akzeptanz der Short Story im Schatten des Romans verweist, wenn sie nämlich aus Joyce' Wahrnehmungsperspektive über Christie O’Dells Short Story Sammlung schreibt:

How Are We to Live is the book's title. A collection of short stories, not a novel. This in itself is a disappointment. It seems to diminish the book's authority, making the author seem like somebody who is just hanging on to the gates of Literature, rather than safely settled inside. (Munro 2007, 49-50)

Es ist eine weitere Ironie, dass Munro selber mit grandiosen Erzählungen wie "Fiction" darauf hingewirkt hat, dass derartige gattungshierarchisierende, ja gattungsdiskriminierende Passagen wie die selbstironische zitierte fortan endgültig der Vergangenheit angehören sollten: Denn, um den Rahmen zu schließen, die weltweit höchste literarische Auszeichnung ging 2013 nicht nur an Alice Munro und nicht nur nach Kanada, sondern ähnlich überfälligerweise auch an nicht nur Alice Munros Lieblingsgattung, die Short Story.

\section{Angeführte Literatur}

Beran, Carol (1999). “The Luxury of Excellence: Alice Munro in the New Yorker.” Robert Thacker, ed. The Rest of the Story: Critical Essays on Alice Munro. Toronto: ECW Press, 204-232.

Berndt, Kathrin (2010). "The Ordinary Terrors of Survival: Alice Munro and the Canadian Gothic." Journal of the Short Story in English 55. Special issue on "The Short Stories of Alice Munro," guest ed. Héliane Ventura, 19-33.

CBC News (2013). "Alice Munro is 1st Canadian Woman to Win Nobel Literature Prize." CBC News (October 10). <http://www.cbc.ca/news/arts/alice-munro-is-1st-canadian-womanto-win-nobel-literature-prize-1.1958383> (15. Oktober 2014). 
CTV News (2013). “Alice Munro wins Nobel Prize in Literature.” CTV News (October 10). <http:// www.ctvnews.ca/entertainment/alice-munro-wins-nobel-prize-in-literature-a-master-ofthe-short-story-1.1491581> (15. Oktober 2014).

Gibson, Graeme (1973). “Alice Munro.” Eleven Canadian Novelists. Toronto: Anansi, 237-264.

Hancock, Geoff (1987). "Alice Munro." Canadian Writers at Work: Interviews with Geoff Hancock. Toronto: Oxford University Press, 187-224.

Howells, Coral Ann (1990). “Alice Munro's Art of Indeterminacy: The Progress of Love.” Reingard M. Nischik and Barbara Korte, eds. Modes of Narrative: Approaches to American, Canadian and British Fiction. Würzburg: Königshausen \& Neumann, 141-152.

Howells, Coral Ann (2003). “Intimate Dislocations: Alice Munro, Hateship, Friendship, Courtship, Loveship, Marriage.” Contemporary Canadian Women's Fiction: Refiguring Identities. New York: Palgrave Macmillan, 58-73.

McGrath, Charles (2013). “Alice Munro Puts Down Her Pen to Let the World In.” The New York Times. July 1.

Menasse, Eva (2013). “So leicht, als wäre es nichts: Die Erzählungen der Literaturnobelpreisträgerin Alice Munro haben eine Form, die Schriftsteller bewundern.” Die Zeit, Nr. 43. October 17.

Munro, Alice (1960). “The Peace of Utrecht.” The Tamarack Review 15 (Spring 1960), 5-21. repr. in Munro (1968). Dance of the Happy Shades, 190-210.

Munro, Alice (1962). “The Office.” The Montrealer (September), 18-23. repr. in Munro (1968). Dance of the Happy Shades, 59-74.

Munro, Alice (1964). “Boys and Girls.” The Montrealer (December), 25-34. repr. in Munro (1968). Dance of the Happy Shades, 111-127.

Munro, Alice (1968a). Dance of the Happy Shades. Toronto: McGraw-Hill Ryerson.

Munro, Alice (1968b). “Walker Brothers Cowboy.” Dance of the Happy Shades, 1-18.

Munro, Alice (1971a). Lives of Girls and Women. Harmondsworth: Penguin, 1982.

Munro, Alice (1971b). “Epilogue: The Photographer.” Lives of Girls and Women, 239-250.

Munro, Alice (1974a). Something l've Been Meaning to Tell You. Scarborough, ON: Signet, 1975.

Munro, Alice (1974b). “The Ottawa Valley.” Something I've Been Meaning to Tell You, 182-197.

Munro, Alice (1978a). Who Do You Think You Are? Toronto: Macmillan (später auch erschienen unter dem Titel The Beggar Maid (1979), s.u.).

Munro, Alice (1978b). “Simon's Luck.” Munro (1979). Who Do You Think You Are? / Munro (1979). The Beggar Maid: Stories of Flo and Rose, 156-177.

Munro, Alice (1979). The Beggar Maid: Stories of Flo and Rose. Harmondsworth: Penguin, 1980. (vorher auch erschienen unter dem Titel Who Do You Think You Are? (1978), s.o.).

Munro, Alice (1980). “Dulse.” The New Yorker. July 21, 30-39. repr. in Munro (1982). The Moons of Jupiter, 36-59.

Munro, Alice (1982). The Moons of Jupiter. Toronto: Macmillan of Canada.

Munro, Alice (1985). “Lichen.” The New Yorker. July 15, 26-36. repr. in Munro (1986). The Progress of Love, 32-55.

Munro, Alice (1986). The Progress of Love. Toronto: McClelland and Stewart.

Munro, Alice (1990a). Friend of My Youth. Toronto: McClelland and Stewart.

Munro, Alice (1990b). “Friend of My Youth.” The New Yorker. January 22, 36-48. repr. in Munro (1990). Friend of My Youth, 3-26.

Munro, Alice (1998). The Love of a Good Woman. Toronto: McClelland and Stewart.

Munro, Alice (2004). Runaway. Toronto: McClelland and Stewart. 
Munro, Alice (2007). “Fiction.” Harper's Magazine. August. repr. in Munro (2009). Too Much Happiness, 32-61.

Munro, Alice (2009). Too Much Happiness. Toronto: McClelland and Stewart.

Munro, Alice (2011). “Dear Life: A Childhood Visitation.” The New Yorker. September 19. repr. as "Dear Life" in Munro (2012). Dear Life, 299-319.

Munro, Alice (2012). Dear Life. London: Chatto \& Windus.

Nischik, Reingard M. (1987). "The Short Story in Canada: Metcalf and Others Making It New." Die Neueren Sprachen 86: 3/4, 232-246.

Nischik, Reingard M. (2007a). "The Canadian Short Story: Status, Criticism, Historical Survey." Reingard M. Nischik, ed. The Canadian Short Story: Interpretations. Rochester, NY: Camden House, 1-39.

Nischik, Reingard M. (2007b). “(Un-)Doing Gender: Alice Munro, ‘Boys and Girls’ (1964).” Reingard M. Nischik, ed. The Canadian Short Story: Interpretations. Rochester, NY: Camden House, 203-218.

Resnick, Mike (2012). Resnick on the Loose. Rockville, MD: Wildside Press.

Struthers, J.R. (Tim) (1981). “The Real Material: An Interview with Alice Munro.” repr. in Probable Fictions: Alice Munro's Narrative Acts. Louis K. Mackendrick (1983), ed. Downsview, ON: ECW Press, 5-36. 